\title{
A LOCALIZED APPROXIMATION METHOD FOR VORTICAL FLOWS*
}

\author{
R. E. CAFLISCH†, O. F. ORELLANA
}

\begin{abstract}
An approximation method of Moore for Kelvin-Helmholtz instability is formulated as a general method for two-dimensional, incompressible, inviscid flows generated by a vortex sheet. In this method the nonlocal equations describing evolution of the sheet are approximated by a system of (local) differential equations. These equations are useful for predicting singularity formation on the sheet and for analyzing the initial value problem before singularity formation. The general method is applied to a number of problems: Kelvin-Helmholtz instability for periodic vortex sheets, motion of an interface in Hele-Shaw flow, Rayleigh-Taylor instability for stratified flow, and Krasny's desingularized vortex sheet equation. A new physically desingularized vortex sheet equation is proposed, which agrees with the finite thickness vortex layer equations in the localized approximation.
\end{abstract}

Key words. vortex dynamics, free boundaries, Kelvin-Helmholtz, Rayleigh-Taylor, Hele-Shaw

AMS(MOS) subject classification. $76 \mathrm{C} 05$

1. Introduction. For many two-dimensional incompressible, inviscid flows, the vorticity is confined to lie on a vortex sheet, which is a free surface in the flow. The fluid velocity is then determined by the location and strength of the vortex sheet. Such flows include the Kelvin-Helmholtz flow in which the vorticity is present initially, Rayleigh-Taylor flow in which the vorticity is generated baroclinically along a curve of density discontinuity, and motion of a fluid interface in a Hele-Shaw cell for which vorticity is produced by a jump in viscosity across the interface. These problems are described in detail in subsequent sections.

One of the most interesting phenomenon for such free surface flows is singularity formation on the vortex sheet. The singularity may be of weak type, such as infinite curvature of the sheet at a point. These singularities, if they occur, are clearly important to mathematical theory and numerical computation, while their physical significance varies from one problem to the next. For example, for the Kelvin-Helmholtz problem, singularity formation immediately precedes roll-up of the sheet [8], [9]. This in turn contributes to production of small-scale variation in the flow. Analysis of the solution after singularity formation is a major open problem for these free surface flows.

Moore [11], [12] analyzed the formation of singularities for the Kelvin-Helmholtz problem and derived a simple approximate description for the evolution of vortex sheets as a system of differential equations in the circulation variable $\Gamma$ along the sheet. A principal advantage of this approximate description is that it is local in $\Gamma$, in contrast to the nonlocal exact description through the Birkhoff-Rott equation. A second feature of this approximation is that it is hyperbolic in a complex $\Gamma$-direction, so that singularity formation on the sheet can be understood as motion of a singularity in the complex $\Gamma$-plane. The complex plane is purely a mathematical construction with no physical meaning, but when the singularity reaches the real $\Gamma$-line, it actually appears on the

* Received by the editors February 13, 1989; accepted for publication (in revised form) December 20, 1989.

$\dagger$ Department of Mathematics, University of California at Los Angeles. This research was supported in part by Air Force Office of Scientific Research, University Research Initiative grant 86-0352, and by National Science Foundation grant NSF-MCS-83-01260.

¥ Santa Maria University, Valparaiso, Chile. This research was supported in part by Fondo Nacional de Desarrolo Cientifico y Technologico (FONDECYT) grant 235 and Universidad Technica Federico Santa Maria, Valparaiso, Chile.

§ Department of Applied Mathematics, California Institute of Technology, Pasadena, California 91125. 
vortex sheet. Moore's approximate equations were rederived and used by Caflisch and Orellana [4] to prove long-time existence for a slightly perturbed vortex sheet.

Moore's predictions for the singularity time and the type of singularity in the Kelvin-Helmholtz problem have been verified numerically in [1], [8], and [17]. A rigorous mathematical analysis of singularity formation in the Kelvin-Helmholtz problem [5], [6] shows that Moore's approximation (with some modifications) gives a correct description for a large set of singular solutions of weak amplitude. Possible limitations of Moore's approximation and some disagreements between Shelley's numerical results [17] and Moore's analysis are discussed at the ends of $\S \S 2$ and 3 .

The purpose of this paper is to present a general formulation of Moore's approximation method $(\S 2)$, which we call the "localized approximation." In this form it can be applied to a variety of flows involving vortex sheets: the periodic Kelvin-Helmholtz problem $(\S 3)$, an interface in Hele-Shaw flow $(\S 4)$, the Rayleigh-Taylor problem $(\S 5)$, and desingularized vortex sheet equations (\$6) such as that of Krasny [9]. A summary of these results and a discussion of additional applications is presented in the final section, $\S 7$.

2. The localized approximation method. Consider an integral operator $M$ for which the integrand $k$ depends on the independent variable $\Gamma$, as well as on a function $s(\Gamma)$, i.e.,

$$
\begin{aligned}
M & =M[s](\Gamma) \\
& =P V \int k\left(\Gamma, \Gamma^{\prime}, s(\Gamma), s\left(\Gamma^{\prime}\right)\right) d \Gamma^{\prime} .
\end{aligned}
$$

The domain of integration will vary with the application. The integral is taken in the Cauchy principal value sense, because of possible singularities at $\Gamma^{\prime}=\Gamma$ and $\left|\Gamma^{\prime}\right|=\infty$.

We shall assume that $s$ is analytic in $|\operatorname{Im} \Gamma|<\rho$ and that $s$ and $M$ are $2 \pi$-periodic in $\Gamma$. In addition, we assume that

$$
M\left[s_{0}\right] \equiv 0
$$

for any constant $s_{0}$, and as a result that constant terms in $s$ can be ignored. Then if $s$ is regular it can be decomposed as

$$
s=s_{+}+s_{-}
$$

in which $s_{+}=\sum_{1}^{\infty} \hat{s}(k) e^{i k \Gamma}$ is analytic in the upper half plane $\{\operatorname{Im} \Gamma>-\rho\}$ and $s_{-}=$ $\sum_{-1}^{-\infty} \hat{s}(k) e^{i k \Gamma}$ is analytic in the lower half plane $\{\operatorname{Im} \Gamma<\rho\}$. The integral operator $M$ can be written as

$$
M=M\left[s_{+}\right](\Gamma)+M\left[s_{-}\right](\Gamma)+E[s](\Gamma) .
$$

Although (2.4) is just a definition of $E$, we claim that $E$ is negligible compared to the other terms in (2.4). The reason for this can be understood in two equivalent ways.

The first justification for neglecting $E$ uses properties of upper and lower analytic functions. The difference

$$
E[s]=M\left[s_{+}+s_{-}\right]-M\left[s_{+}\right]-M\left[s_{-}\right]
$$

depends only on cross multiplication terms such as $s_{+} s_{-}$, and not on pure $s_{+}$or pure $s_{-}$terms such as $s_{+}^{2}$ or $s_{-}^{2}$. The upper analytic function $s_{+}$is exponentially decaying 
in the $\operatorname{Im}(\Gamma)$ direction, while $s_{-}$is exponentially decaying in the $-\operatorname{Im}(\Gamma)$ direction, i.e., for $\operatorname{Im} \Gamma>-\rho$

$$
\left|s_{+}(\Gamma)\right| \leqq e^{-(\rho+\operatorname{Im}(\Gamma))} \sup _{\operatorname{Im} \Gamma^{\prime}=-\rho}\left|s_{+}\left(\Gamma^{\prime}\right)\right|
$$

and for $\operatorname{Im} \Gamma<\rho$

$$
\left|s_{-}(\Gamma)\right| \leqq e^{-(\rho-\operatorname{Im}(\Gamma))} \sup _{\operatorname{Im} \Gamma^{\prime}=\rho}\left|s_{-}\left(\Gamma^{\prime}\right)\right| .
$$

A detailed proof of (2.6a) and (2.6b) is provided in Appendix A of [4]. As a consequence, for $|\operatorname{Im} \Gamma| \leqq \rho$

$$
\left|s_{+} s_{-}(\Gamma)\right| \leqq e^{-2 \rho}\left(\sup _{\operatorname{Im} \Gamma^{\prime}=-\rho}\left|s_{+}\left(\Gamma^{\prime}\right)\right|\right)\left(\sup _{\operatorname{Im} \Gamma^{\prime}=\rho}\left|s_{-}\left(\Gamma^{\prime}\right)\right|\right) .
$$

Define $\|s\|=\sup _{|\operatorname{Im} \Gamma| \leqq \rho}|s(\Gamma)|$. Then (2.7) says that $\left\|s_{+} s_{-}\right\| \leqq e^{-2 \rho}\left\|s_{+}\right\|\left\|s_{-}\right\|$. If $M$ is a good operator, it follows that

$$
\|E[s]\| \leqq e^{-2 \rho}\left\|s_{+}\right\|\left\|s_{-}\right\|,
$$

whereas $M\left[s_{ \pm}\right]=O\left(\left\|s_{ \pm}\right\|\right)$. Therefore if $\rho \gg 1, E[s]$ is negligible.

A second explanation for the smallness of $E$ comes from looking at the Fourier series for $s$. This is the original explanation of Moore [11] and is actually equivalent to the first explanation. Assume that $s$ has the form

$$
s(\Gamma)=\sum_{\substack{k=-\infty \\ k \neq 0}}^{\infty} \varepsilon^{|k|} a_{k} e^{i k \Gamma} .
$$

This is equivalent to the assumption that $s$ is analytic in $\{|\operatorname{Im} \Gamma|<\rho\}$ with $\rho=|\log \varepsilon|$. Such an expansion is natural if $s$ is originally of the form $s=\varepsilon a_{1} e^{i \Gamma}+\varepsilon a_{-1} e^{-i \Gamma}$ (or any finite number of wavenumbers) and $s$ evolves in time due to a nonlinear process. Then higher wavenumbers are generated by wavenumber doubling through multiplication of $s$ with itself, so that the higher wavenumbers come with geometrically decreasing amplitudes. Now consider the product $s^{2}$ which is expanded as

$$
\begin{aligned}
s^{2}(\Gamma) & =\sum_{\substack{k_{1}=-\infty \\
k_{1} \neq 0}}^{\infty} \sum_{\substack{k_{2}=-\infty \\
k_{2} \neq 0}}^{\infty} \varepsilon^{\left|k_{1}\right|+\left|k_{2}\right|} a_{k_{1}} a_{k_{2}} e^{i\left(k_{1}+k_{2}\right) \Gamma} \\
& =\sum_{\operatorname{sgn}\left(k_{1}\right)=\operatorname{sgn}\left(k_{2}\right)}\left(\varepsilon^{\left|k_{1}+k_{2}\right|} a_{k_{1}} a_{k_{2}}+O\left(\varepsilon^{\left|k_{1}+k_{2}\right|+1}\right)\right) e^{i\left(k_{1}+k_{2}\right) \Gamma} .
\end{aligned}
$$

Since $\left|k_{1}\right|+\left|k_{2}\right|=\left|k_{1}+k_{2}\right|$ only if $\operatorname{sgn}\left(k_{1}\right)=\operatorname{sgn}\left(k_{2}\right)$, only products of two positive $k$ terms or two negative $k$ terms are significant, i.e., only $s_{+}^{2}$ and $s_{-}^{2}$ terms. For all cross multiplications of negative $k$ and positive $k$ terms, the wavenumber sum $k_{1}+k_{2}$ involves cancellation while the amplitude exponent $\left|k_{1}\right|+\left|k_{2}\right|$ add. Since $E[s]$ involves only products of + and - terms, it is negligible.

Return now to equation (2.4). Since $E[s]$ is negligible, $M$ can be approximated by

$$
M[s] \simeq M\left[s_{+}\right]+M\left[s_{-}\right] .
$$

The advantage of the approximation (2.11) over the original operator (2.1) is that the two integral operators on the right of (2.11) involve an integrand that is analytic in the upper or lower half planes. Thus we can hope to directly evaluate them by appropriate contour deformation.

Now suppose that $s$ evolves in time according to the integrodifferential equation

$$
\partial_{t} s^{*}(\Gamma, t)=M[s](\Gamma, t)
$$


in which $s^{*}(\Gamma)=\overline{s(\bar{\Gamma})}$ and the overbar indicates the usual complex conjugate. The function $s^{*}$ is the analytic extension of the conjugate function $\bar{s}$ from the real $\Gamma$ line. Define the projection $H_{+}$onto the space of upper analytic functions and the projection $H_{-}$onto that of lower analytic functions. Since the operator ${ }^{*}$ switches upper and lower half planes,

$$
\begin{aligned}
& H_{+}\left(s^{*}\right)=\left(H_{-} s\right)^{*}=\left(s_{-}\right)^{*}, \\
& H_{-}\left(s^{*}\right)=\left(H_{+} s\right)^{*}=\left(s_{+}\right)^{*} .
\end{aligned}
$$

We also assume that $M\left[s_{+}\right]$is upper analytic and $M\left[s_{-}\right]$is lower analytic, i.e., that

$$
\begin{aligned}
& H_{+} M\left[s_{+}\right]=M\left[s_{+}\right], \\
& H_{-} M\left[s_{-}\right]=M\left[s_{-}\right] .
\end{aligned}
$$

Now use the localized approximation (2.11) in (2.12), with $s=s_{+}+s_{-}$, to obtain

$$
\partial_{t}\left(s_{+}\right)^{*}+\partial_{t}\left(s_{-}\right)^{*}=M\left[s_{+}\right]+M\left[s_{-}\right] .
$$

Apply the operators $H_{+}$and $H_{-}$to (2.15) using the relations (2.13) and (2.14) to obtain

$$
\begin{aligned}
& \partial_{t}\left(s_{+}\right)^{*}=M\left[s_{-}\right], \\
& \partial_{t}\left(s_{-}\right)^{*}=M\left[s_{+}\right] .
\end{aligned}
$$

It will be more convenient to have equations for $s_{+}$and $\left(s_{-}\right)^{*}$, both of which are analytic in the upper half plane. These are obtained by applying * to $(2.16)$ to get the equations

$$
\begin{aligned}
& \partial_{t} s_{+}=M\left[s_{-}\right]^{*}, \\
& \partial_{t} s_{-}^{*}=M\left[s_{+}\right]
\end{aligned}
$$

in which $s_{-}^{*}=\left(s_{-}\right)^{*}$. The system $(2.17)$ is the localized approximation for the evolution equation (2.12).

A similar localized approximation can be made if $M[s]$ is replaced by the somewhat more complicated operator $M\left[s, s^{*}\right](\Gamma, t)$. Since $M$ depends on both $s$ and $s^{*}$, the localized approximation (2.11) for $M$ must be modified. The functions $s_{+}$and $s_{-}^{*}=\left(s_{-}\right)^{*}$ are upper analytic, while $s_{-}$and $s_{+}^{*}=\left(s_{+}\right)^{*}$ are lower analytic. As before, product terms $s_{+} s_{-}, s_{-} s_{+}^{*}, s_{-} s_{-}^{*}$, and $s_{+}^{*} s_{-}^{*}$ are negligible compared to the purely upper analytic or purely lower analytic terms $s_{+}^{2}, s_{-}^{2}, s_{+}^{* 2}, s_{+} s_{-}^{*}, s_{-} s_{+}^{*}$, and $s_{-}^{* 2}$. This leads to the localized approximation

$$
M\left[s, s^{*}\right] \cong M\left[s_{+}, s_{-}^{*}\right]+M\left[s_{-}, s_{+}^{*}\right]
$$

for $M$.

Now consider an evolution equation of the form

$$
\partial_{t} s^{*}=M\left[s, s^{*}\right] .
$$

Use of the approximation (2.18), projection by $H_{+}$and $H_{-}$, and application of * to the $H_{-}$equation results in the localized approximation

$$
\begin{aligned}
& \partial_{t} s_{+}=M\left[s_{-}, s_{+}^{*}\right]^{*}, \\
& \partial_{t} s_{-}^{*}=M\left[s_{+}, s_{-}^{*}\right]
\end{aligned}
$$

in which $s_{-}^{*}=\left(s_{-}\right)^{*}$ and $s_{+}^{*}=\left(s_{+}\right)^{*}$.

The localized approximations (2.17) or (2.20) for the evolution equations (2.12) or (2.19), respectively, are the main results of this section. We shall employ this 
approximation and examine its consequences for a variety of problems below. For these applications we shall find that $M\left[s_{+}\right]$and $M\left[s_{-}\right]^{*}\left(\right.$ or $M\left[s_{+}, s_{-}^{*}\right]$ and $\left.M\left[s_{--}, s_{+}^{*}\right]^{*}\right)$ can be explicitly evaluated as differential operators.

Note that the localized approximation contains all of the linear terms and thus has the same linearization as the original problem. For singularities far from the real axis, it also contains the most important nonlinear terms. Thus the approximation should correctly describe both the nonlinear production of singularities far from the real axis and the propagation of singularities toward the real axis. If the initial perturbation $s$ is of size $\varepsilon$, then it is expected to take time $\log \left(\varepsilon^{-1}\right)$ for the singularities to reach the real axis [11], [12]. The bound (2.8) suffices to make $E[s]$ negligible even over this long time period [4].

Once the singularities are near the real axis, then $\rho$ (the width of the strip of analyticity) is not large, and the factor $e^{-2 \rho}$ in the bound (2.8) is not small. However if the perturbation $s$ in the vortex sheet position is small at the singularity time (as in the Kelvin-Helmholtz problem), then the nonlinear term $E[s]$ will still be negligible. In this case the localized approximation should correctly describe the singularity formation. Even if the vortex sheet perturbations are large at the singularity time, so that the localized approximation is not quantitatively accurate, we still expect that it will give a reasonable estimate of both the singularity time and the type of singularity. As described at the end of $\S 5$, this is the case for the Boussinesq limit of the Rayleigh-Taylor problem. Thus we expect the localized approximation to give a valid description of singularity formation in free surface flows.

3. The Kelvin-Helmholtz problem. The Kelvin-Helmholtz problem is to describe the evolution of a small periodic perturbation of a flat vortex sheet of uniform strength. The location of the sheet is described by a complex function $z(\Gamma, t)=x+i y$, in which the real variable $\Gamma$ parameterizes the sheet. Choose the parameterization $\Gamma$, so that $\partial \Gamma / \partial s=$ vorticity density along the sheet. The evolution of the sheet is governed by the Birkhoff-Rott equation [3]

$$
\frac{\partial z^{*}}{\partial t}=(2 \pi i)^{-1} P V \int_{-\infty}^{\infty}\left(z(\Gamma)-z\left(\Gamma^{\prime}\right)\right)^{-1} d \Gamma^{\prime}
$$

for $\Gamma$ real. A special equilibrium solution of (3.1) is $z=\Gamma$, corresponding to a flat vortex sheet of uniform strength.

Write $z$ as a perturbation of this exact solution, i.e.,

$$
z(\Gamma, t)=\Gamma+s(\Gamma, t)
$$

and define the integral operator

$$
B[s](\Gamma)=(2 \pi i)^{-1} P V \int_{-\infty}^{\infty}\left(\Gamma-\Gamma^{\prime}+s(\Gamma)-s\left(\Gamma^{\prime}\right)\right)^{-1} d \Gamma^{\prime} .
$$

In (3.3) constants in $s$ do not matter, so that we may assume that $s_{0}=0$. The operator $B[s]$ is of the form considered in $\S 2$. Then Moore's approximation (i.e., the localized approximation) for $B$ is

$$
\begin{aligned}
B[s] & \simeq B\left[s_{+}\right]+B\left[s_{-}\right] \\
& =\frac{1}{2} \frac{\partial_{\Gamma} s_{+}}{1+\partial_{\Gamma} s_{+}}-\frac{1}{2} \frac{\partial_{\Gamma} s_{-}}{1+\partial_{\Gamma} s_{-}} .
\end{aligned}
$$

The second line of (3.4) comes from the exact evaluations $B\left[s_{+}\right]=\frac{1}{2}\left(\partial_{\Gamma} s_{+}\right)\left(1+\partial_{\Gamma} s_{+}\right)^{-1}$ and $B\left[s_{-}\right]=-\frac{1}{2}\left(\partial_{\Gamma} s_{-}\right)\left(1+\partial_{\Gamma} s_{-}\right)^{-1}$, details of which are in [4]. If we assume that $\left|\partial_{\Gamma} s_{ \pm}\right|<1$, 
then $B\left[s_{+}\right]=\frac{1}{2} \partial_{\Gamma} s_{+} /\left(1+\partial_{\Gamma} s_{+}\right)$and $B\left[s_{-}\right]^{*}=-\frac{1}{2} \partial_{\Gamma} s_{-}^{*} /\left(1+\partial_{\Gamma} s_{-}^{*}\right)$ are upper analytic, as assumed in $\S 2$. Then the localized approximation (2.17) for the Birkhoff-Rott equation (3.1) is

$$
\begin{aligned}
& \partial_{t} s_{-}^{*}=\frac{1}{2} \frac{\partial_{\Gamma} s_{+}}{1+\partial_{\Gamma} s_{+}}, \\
& \partial_{t_{+}} s_{+}=-\frac{1}{2} \frac{\partial_{\Gamma} s_{-}^{*}}{1+\partial_{\Gamma} s_{-}^{*}}
\end{aligned}
$$

in which $s_{-}^{*}=\left(s_{-}\right)^{*}$.

The system (3.5) can be made quasi-linear by differentiation in $\Gamma$, with the definition $\phi=\partial_{\Gamma} s_{+}, \psi=\partial_{\Gamma} s_{-}^{*}$, to obtain

$$
\begin{aligned}
& \partial_{t} \psi=-\frac{1}{2} \partial_{\Gamma}(1+\phi)^{-1}, \\
& \partial_{t} \phi=\frac{1}{2} \partial_{\Gamma}(1+\psi)^{-1} .
\end{aligned}
$$

Equations (3.6) are the localized approximation for the Kelvin-Helmholtz problem and are the main goal of this section.

The system (3.6) is a nonlinear hyperbolic system in complex $\Gamma$ versus $t$ with Riemann invariants

$$
\begin{aligned}
& r=\log (1+\phi)+i \log (1+\psi) \\
& s=\log (1+\phi)-i \log (1+\psi)
\end{aligned}
$$

and characteristic speeds

$$
\begin{aligned}
& \lambda=-\frac{i}{2}(1+\phi)^{-1}(1+\psi)^{-1}, \\
& \mu=\frac{i}{2}(1+\phi)^{-1}(1+\psi)^{-1}
\end{aligned}
$$

satisfying

$$
\begin{aligned}
& r_{t}+\lambda r_{\Gamma}=0, \\
& s_{t}+\mu s_{\Gamma}=0 .
\end{aligned}
$$

Moore [9], [10] used the Riemann invariant form for this system (actually he used an equivalent system (3.11) below) to describe singularity formation for the vortex sheet. This application of nonlinear hyperbolic equations is unusual in that the physically correct singularity occurs as an envelope of the characteristics. The reason is that the characteristics move approximately in the imaginary $\Gamma$ direction, whereas the physical line is the real $\Gamma$ axis. As sketched in Fig. 1 an envelope for the characteristics may form off in the complex plane and travel to the real line. Up until the time that it hits the real axis, the solution on the real axis is smooth. At the envelope, the generic behavior of $\phi$ and $\psi$ is $\left(\Gamma-\Gamma_{e}\right)^{1 / 2}$, so that the generic singularity for the vortex sheet is $z \simeq\left(\Gamma-\Gamma_{e}\right)^{3 / 2}$ (since $\phi$ and $\psi$ are related to $z_{\Gamma}$ ). At the time of physical appearance of the singularity on the real $\Gamma$ line, two such $\frac{3}{2}$ singularities may collide.

Note that after the envelope forms (say on the imaginary axis), the solution cannot be continued past the envelope. Actually, as shown in Fig. 2, there are two envelope curves. Continuation of the solution from the real axis is stopped by the envelope curve $E_{1}$. A shock could be inserted into the solution to make a jump from the sheet $S_{1}$ onto the sheet $S_{2}$ (cf. Fig. 3), if the solution needs to be continued to the entire 


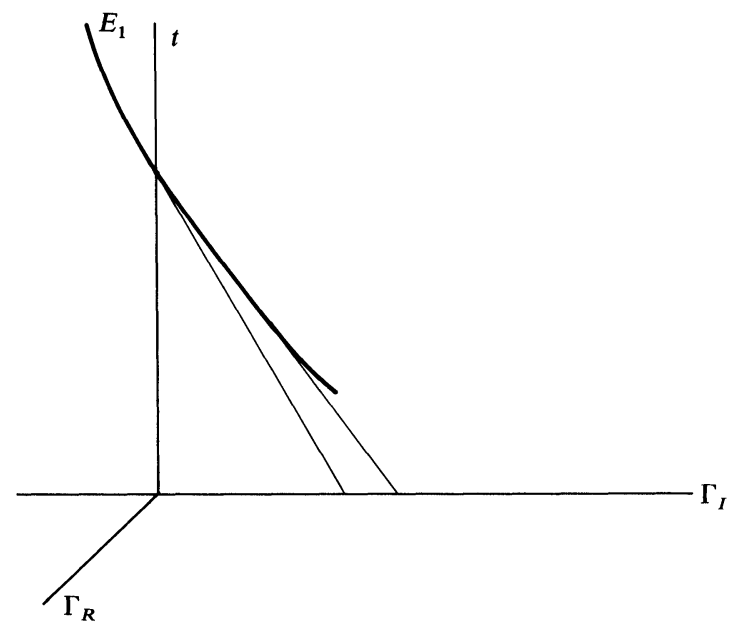

FIG. 1. One family of characteristics and their envelope for the system (3.12). A smooth solution on the physical line $\Gamma_{R}$ (i.e., $\Gamma$ real) exists until time $T$. The characteristics have been drawn as straight lines for simplicity.

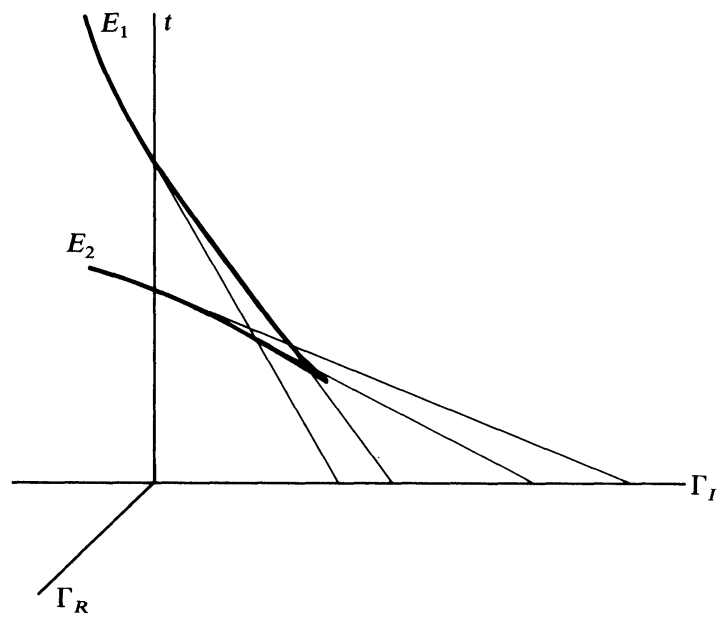

FIG. 2. Continuation of Fig. 1 to all $\Gamma_{I}$. At initial formation of the envelope it has two branches. Only the upper one is needed here, as in Fig. 1. A shock $(s)$ would be inserted for a gas dynamics problem.

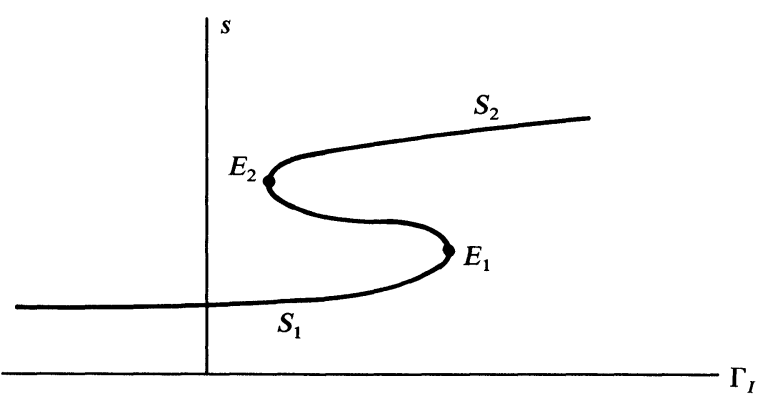

FIG. 3. The solution as a multivalued function. A smooth solution exists on the lower branch $S_{1}$ up to the envelope $E_{1}$. 
imaginary axis. However, since the real axis is the physical line, this is unnecessary (in contrast to usual applications of hyperbolic equation), so that no shock is needed.

The system used by Moore in [11] and [12] is a transformation of (3.6). Set

$$
\phi=-1+(1 / \sqrt{2}) h^{1 / 2} e^{-i g / 2}
$$

and set $y=i \Gamma$. Then $g$ and $h$ satisfy

$$
\begin{aligned}
& h_{t}=g_{y}, \\
& g_{t}=h^{-2} h_{y},
\end{aligned}
$$

which is the system obtained by Moore [12]. The localized approximation equations (3.6) or (3.11) were also used by Caflisch and Orellana [4] to prove long-time existence for $s$ that is initially small and analytic in a wide strip $\{|\operatorname{Im} \Gamma|<\rho\}$.

Recent computations by Shelley [17] confirm the $\frac{3}{2}$ power singularity and Moore's expression for the singularity time. However for initial perturbations of moderate size, they show some disagreement with Moore's expression for the form of the singularity. We believe that Moore's approximate equations (3.11) would give the correct form for the singularity, and that these discrepancies come from Moore's asymptotic solution of equations (3.11). This possibility is currently being tested.

4. Interfaces in Hele-Shaw flow. Flow in a narrow gap between two plates is described by the Hele-Shaw equations. The interface between two fluids in a Hele-Shaw cell is a vortex sheet, since the tangential velocity is discontinuous there. Trygvasson and Aref [19] show how to describe the dynamics of such an interface by an integraldifferential equation on the interface. We first recall this integral-differential equation, then rewrite it in the complex analytic notation of the previous section, so that the localized approximation method of $\S 2$ can be applied.

The Hele-Shaw equations are

$$
\begin{aligned}
& \nabla p=-\left(\mu_{1} / k_{1}\right) \mathbf{u}+\rho_{1} \mathbf{g}, \\
& \nabla \cdot \mathbf{u}=0 \quad \text { in } \Omega_{1}, \\
& \nabla p=-\left(\mu_{2} / k_{2}\right) \mathbf{u}+\rho_{2} \mathbf{g}, \\
& \nabla \cdot \mathbf{u}=0 \quad \text { in } \Omega_{2}
\end{aligned}
$$

for upper fluid 1 in $\Omega_{1}$ and lower fluid 2 in $\Omega_{2}$ with fluid interface $S=\partial \Omega_{1} \cap \partial \Omega_{2}$. The forcing has been assumed to be gravitational, but this is equivalent to a prescribed pressure gradiant. The boundary conditions across $S$ are

$$
\begin{aligned}
& p_{1}=p_{2}, \\
& \mathbf{n} \cdot \mathbf{u}_{1}=\mathbf{n} \cdot \mathbf{u}_{2}
\end{aligned}
$$

since there is no surface tension. At infinity the velocities $\mathbf{u}_{1}$ and $\mathbf{u}_{2}$ are assumed to go to zero. The vorticity density $\gamma$ on $S$ is found to be

$$
\begin{aligned}
\gamma & \equiv\left(\mathbf{u}_{2}-\mathbf{u}_{1}\right) \cdot \tau \\
& =2 a \mathbf{U}_{a} \cdot \tau-2 b \hat{\mathbf{g}} \cdot \tau
\end{aligned}
$$

in which $\mathbf{U}_{a}=\left(\mathbf{u}_{1}+\mathbf{u}_{2}\right) / 2$ is the average velocity of the two fluids at the interface and

$$
\begin{gathered}
a=\left(\mu_{1} / k_{1}-\mu_{2} / k_{2}\right) /\left(\mu_{1} / k_{1}+\mu_{2} / k_{2}\right), \\
b=\left(\rho_{1}-\rho_{2}\right)|g| /\left(\mu_{1} / k_{1}+\mu_{2} / k_{2}\right), \\
\hat{\mathbf{g}}=\mathbf{g} /|g|=(0,-1) .
\end{gathered}
$$


For the interface velocity $\mathbf{U}=\partial \mathbf{x} / \partial t$ we will use a weighted average of the two fluid velocities at the interface, i.e.,

$$
\mathbf{U}=\frac{1-\beta}{2} \mathbf{u}_{1}+\frac{1+\beta}{2} \mathbf{u}_{2}
$$

in which $-1 \leqq \beta \leqq 1$ will be chosen later. Note that this choice is permissible since it has the correct normal component $\mathbf{n} \cdot \mathbf{U}=\mathbf{n} \cdot \mathbf{u}_{1}=\mathbf{n} \cdot \mathbf{u}_{2}$, while the tangential component for the velocity of any interface is arbitrary. Such a weighted average seems desirable, because for an interface between liquid and gas with $\rho_{2}=\mu_{2}=0$, the most reasonable choice for $\mathbf{U}$ is $\mathbf{U}=\mathbf{u}_{1}$. The equation for motion of the interface is then

$$
\partial_{t} \mathbf{x}(\Gamma, t)=\mathbf{U}_{a}+\frac{\beta}{2} \gamma \tau
$$

Parameterize the interface as $\mathbf{x}(\Gamma)$. The Biot-Savart law says that the average velocity $\mathbf{U}_{a}$ is

$$
\begin{aligned}
\mathbf{U}_{a} & =(2 \pi)^{-1} P V \int_{-\infty}^{\infty} \frac{\hat{\mathbf{z}} \times\left(\mathbf{x}(\Gamma)-\mathbf{x}\left(\Gamma^{\prime}\right)\right)}{\left|\mathbf{x}(\Gamma)-\mathbf{x}\left(\Gamma^{\prime}\right)\right|^{2}} \gamma\left(\Gamma^{\prime}\right) d s\left(\Gamma^{\prime}\right) \\
& =(2 \pi)^{-1} P V \int_{-\infty}^{\infty} \frac{\hat{\mathbf{z}} \times\left(\mathbf{x}(\Gamma)-\mathbf{x}\left(\Gamma^{\prime}\right)\right)}{\left|\mathbf{x}(\Gamma)-\mathbf{x}\left(\Gamma^{\prime}\right)\right|^{2}} \gamma\left(\Gamma^{\prime}\right) \frac{\partial s}{\partial \Gamma^{\prime}} d \Gamma^{\prime} .
\end{aligned}
$$

Equations (4.3), (4.5), and (4.6) give a complete description of the interface motion. They can be rewritten in complex variable notation by defining $z(\Gamma)=x(\Gamma)+i y(\Gamma)$, $v=\gamma\left(\tau_{x}+i \tau_{y}\right)$, and $w=U_{a}+i V_{a}$. The vorticity density $\gamma(\partial s / \partial \Gamma)$ is then found to be

$$
\begin{aligned}
\gamma \frac{\partial s}{\partial \Gamma} & =2\left(a \mathbf{U}_{a}-b \mathbf{g}\right) \cdot\left(\tau \frac{\partial s}{\partial \Gamma}\right) \\
& =2\left(a U_{a}, a V_{a}+b\right) \cdot\left(x_{\Gamma}, y_{\Gamma}\right) \\
& =2 a\left(U_{a} x_{\Gamma}+V_{a} y_{\Gamma}\right)+2 b y_{\Gamma} \\
& =a\left(\bar{w} z_{\Gamma}+w \bar{z}_{\Gamma}\right)-i b\left(z_{\Gamma}-\bar{z}_{\Gamma}\right) \\
& =a\left(w^{*} z_{\Gamma}+w z_{\Gamma}^{*}\right)-i b\left(z_{\Gamma}-z_{\Gamma}^{*}\right) .
\end{aligned}
$$

Equality (4.7) is for real $\Gamma$. Replacement of $\bar{z}$ by $z^{*}$ (the analytic extension of $\bar{z}$ ) is valid for real $\Gamma$ and analytically extends the formula for $\sigma(\partial s / \partial \Gamma)$ to complex $\Gamma$. Similarly,

$$
\begin{gathered}
v^{*}=z_{\Gamma}^{-1}\left\{a\left(w^{*} z_{\Gamma}+w z_{\Gamma}^{*}\right)-i b\left(z_{\Gamma}-z_{\Gamma}^{*}\right)\right\}, \\
w^{*}=H\left[z, z^{*}\right]=(2 \pi i)^{-1} P V \int_{\infty}^{\infty}\left(z-z^{\prime}\right)^{-1}\left\{a\left(w^{* \prime} z_{\Gamma}^{\prime}+w^{\prime} z_{\Gamma}^{* \prime}\right)-i b\left(z_{\Gamma}^{\prime}-z_{\Gamma}^{* \prime}\right)\right\} d \Gamma^{\prime}
\end{gathered}
$$

with $z=z(\Gamma), z^{\prime}=z\left(\Gamma^{\prime}\right)$, etc., on the right side. Finally, (4.5) for the motion of the interface is equivalent to

$$
\partial_{t} z^{*}(\Gamma, t)=w^{*}+\frac{\beta}{2} v^{*} .
$$

An obvious solution of (4.9) is $z \equiv \Gamma$ with $w \equiv 0$.

The complex analytic equation (4.9) can now be analyzed using the localized approximation method. Look for $z$ to be a slight perturbation of the steady-state solution $z_{0}=\Gamma$, i.e.,

$$
\begin{aligned}
z & =\Gamma+s(\Gamma, t) \\
& =\Gamma+s_{+}(\Gamma, t)+s_{-}(\Gamma, t)
\end{aligned}
$$


and denote

$$
M\left[s, s^{*}\right]=H\left[z, z^{*}\right] .
$$

The localized approximation for $M$, according to (2.18), is

$$
M\left[s, s^{*}\right]=M\left[s_{+}, s_{-}^{*}\right]+M\left[s_{-}, s_{+}^{*}\right]
$$

in which

$$
\begin{aligned}
& M\left[s_{+}, s_{-}^{*}\right](\Gamma) \\
& =(2 \pi i)^{-1} P V \int_{-\infty}^{\infty}\left(z_{+}-z_{+}^{\prime}\right)^{-1} \\
& \quad \cdot\left\{a w_{-}^{* \prime} z_{+\Gamma}^{\prime}+a w_{+}^{\prime} z_{-\Gamma}^{* \prime}-i b\left(z_{+\Gamma}^{\prime}-z_{-\Gamma}^{* \prime}\right)\right\} d \Gamma^{\prime},
\end{aligned}
$$

where we denote $z_{+}=\Gamma+s_{+}, z_{-}=\Gamma+s_{-}$, etc. A straightforward contour integration shows that

$$
\begin{aligned}
M\left[s_{+}, s_{-}^{*}\right] & =-\frac{1}{2} z_{+\Gamma}^{-1}\left\{a w_{-}^{*} z_{+\Gamma}+a w_{+} z_{-\Gamma}^{*}-i b\left(z_{+\Gamma}-z_{-\Gamma}^{*}\right)\right\} \\
M\left[s_{-}, s_{+}^{*}\right]^{*} & =\frac{1}{2} z_{-\Gamma}^{*-1}\left\{a w_{+} z_{-\Gamma}^{*}+a w_{-}^{*} z_{+\Gamma}+i b\left(z_{-\Gamma}^{*}-z_{+\Gamma}\right)\right\} \\
& =-z_{-\Gamma}^{*-1} z_{+\Gamma} M\left[s_{+}, s_{-}^{*}\right] .
\end{aligned}
$$

The change in sign in (4.14b) comes because the integration contour is deformed downwards, rather than upwards as in (4.14a). If $\left|s_{ \pm \Gamma}\right|<\frac{1}{2}$, then $M\left[s_{+}, s_{-}^{*}\right]$ and $M\left[s_{-}, s_{+}^{*}\right]^{*}$ are upper analytic, as required.

The localized approximation for $w$ is (after some cancellation)

$$
\begin{aligned}
& w_{-}^{*}=M\left[s_{+}, s_{-}^{*}\right]=\frac{i b}{2} \frac{s_{+\Gamma}-s_{-\Gamma}^{*}}{1+s_{+\Gamma}}, \\
& w_{+}=M\left[s_{-}, s_{+}^{*}\right]^{*}=\frac{-i b}{2} \frac{s_{+\Gamma}-s_{-\Gamma}^{*}}{1+s_{-\Gamma}^{*}} .
\end{aligned}
$$

Similarly, the localized approximation for $v$ is

$$
\begin{aligned}
v_{-}^{*} & =z_{+\Gamma}^{-1}\left\{a\left(w_{-}^{*} z_{+\Gamma}+w_{+} z_{-\Gamma}^{*}\right)-i b\left(z_{+\Gamma}-z_{-\Gamma}^{*}\right)\right\} \\
& =i b\left(s_{-\Gamma}^{*}-s_{+\Gamma}^{*}\right) /\left(1+s_{+\Gamma}\right), \\
v_{+} & =z_{-\Gamma}^{*-1}\left\{a\left(w_{+} z_{-\Gamma}^{*}+w_{-}^{*} z_{+\Gamma}\right)+i b\left(z_{-\Gamma}^{*}-z_{+\Gamma}\right)\right\} \\
& =i b\left(s_{-\Gamma}^{*}-s_{+\Gamma}^{*}\right) /\left(1+s_{-\Gamma}^{*}\right) .
\end{aligned}
$$

The localized approximation (2.20) for the Hele-Shaw equation (4.5) is then

$$
\begin{aligned}
& \partial_{t} s_{+}=w_{+}+\frac{\beta}{2} v_{+}, \\
& \partial_{t} s_{-}^{*}=w_{-}^{*}+\frac{\beta}{2} v_{-}^{*},
\end{aligned}
$$

i.e.,

$$
\begin{aligned}
& s_{+t}=\frac{i b}{2}(1+\beta) \frac{s_{-\Gamma}^{*}-s_{+\Gamma}}{1+s_{-\Gamma}^{*}}, \\
& s_{-t}^{*}=-\frac{i b}{2}(1-\beta) \frac{s_{-\Gamma}^{*}-s_{+\Gamma}}{1+s_{+\Gamma}} .
\end{aligned}
$$


Equations (4.18) can be cast into a very simple form by differentiating them in $\Gamma$ and defining

$$
\begin{aligned}
& u=\frac{1-\beta}{1+s_{+\Gamma}}+\frac{1+\beta}{1+s_{-\Gamma}^{*}}, \\
& v=\frac{1+s_{+\Gamma}}{1+s_{-\Gamma}^{*}} .
\end{aligned}
$$

Then (4.18) is equivalent to

$$
\begin{aligned}
& \partial_{t} u=0, \\
& \partial_{t} v+\frac{i b}{2} u \partial_{\Gamma} v=0,
\end{aligned}
$$

which is a linearly degenerate hyperbolic system. The system (4.20) is the localized approximation for an interface in a Hele-Shaw cell. Singularities occur in the solution of this system only if they are present initially somewhere in the complex plane. They propagate along the characteristics $d z / d t=0$ or $d z / d t=u(z)$.

At equilibrium $z=\Gamma$, the quantities $u$ and $v$ are $u=2$ and $v=1$. Thus the linearization of (4.20) for $u=2+u^{\prime}, v=1+v^{\prime}$ is

$$
\begin{aligned}
& u_{t}^{\prime}=0, \\
& v_{t}^{\prime}+i b v_{\Gamma}^{\prime}=0,
\end{aligned}
$$

which has the solution $v^{\prime}=e^{i k \Gamma+b k t}$. Since $v$ is upper analytic, $k>0$. Thus there is instability if $b>0$ and stability if $b<0$, in agreement with the linear analysis of Saffman and Taylor [16].

In the extreme case of an interface between a liquid and a gas, i.e., $\rho_{2}=\mu_{2}=0$, it is natural to take the interface velocity to be the velocity of the liquid, which means to set $\beta=-1$. For this special case, there is a simple exact set of equations, which differ from the localized approximation equations (4.18). The simplified system is obtained by parameterizing the interface as $z(\kappa, t)=\kappa+h(\kappa, t)$ in which $h$ is upper analytic in $\kappa$. The $\kappa$-parameterization, which is determined by the requirement of upper analyticity, differs from the $\Gamma$-parameterization above, which is determined by the velocity definition (4.5).

Using this simplified system for the liquid-gas interface, exact singular solutions have been derived by Howison, Ockendon, and Lacey [7] and exact smooth solutions by Saffman [15]. For the singular solutions of Howison, Ockendon, and Lacey [7] the singular point is $\kappa=i \kappa_{0}$ at which $\partial_{\kappa} z=0$. This can be transformed to a point at which $\partial_{\Gamma}^{2} z=\infty$, by the mapping $\Gamma=\kappa+a \cos \kappa$ at $t=0$ with proper choice of $a$ so that $\partial_{\kappa} \Gamma\left(i \kappa_{0}\right)=0$. Moreover, if $\left|\kappa_{0}\right|$ is large enough, $\Gamma(\kappa)$ is one to one and onto on the interval $[0,2 \pi]$. This choice is more natural for the hyperbolic equation (4.17), since the singular point $\partial_{\Gamma}^{2} z=\infty$ will move along the characteristics until it reaches the physical line $\Gamma$ real. This shows that the singular solutions of [7] correspond to singular solutions of our localized approximation (4.20).

5. The Rayleigh-Taylor problem. The Rayleigh-Taylor problem describes motion of an interface between incompressible, inviscid fluids with different densities. The baroclinic generation of vorticity along this interface makes it into a vortex sheet, which is then subject to instability, singularity formation, and roll-up. As shown in [1], the interface position $z(\alpha, t)$ is governed by a system of integrodifferential equations on the interface (for consistency with [18] the variable $\Gamma$ is replaced by $\alpha$ in this 
section). Neglect surface tension, take the gravitational force to be $(0,-g)$, and let $\gamma(\alpha, t)$ denote the vorticity density on the interface. As in the previous section, we take the vortex sheet velocity $\mathbf{U}$ to be the combination of the upper and lower fluid velocities given by (4.4). We also assume that the zeroth Fourier coefficient $\gamma_{0}$ in $\gamma$ is zero. The equations for the Rayleigh-Taylor problem are then

$$
\begin{aligned}
& \partial_{t} z^{*}(\alpha, t)= R_{1}[z, \gamma] \equiv q^{*}+\frac{\beta \gamma}{2 z_{\alpha}} \\
& \partial_{t} \gamma(\alpha, t)=R_{2}\left[z, z^{*}, \gamma\right] \\
& \equiv A\left[\left(q_{t}^{*} z_{\alpha}+q_{t} z_{\alpha}^{*}\right)+\frac{1}{4} \partial_{\alpha} \frac{\gamma^{2}}{z_{\alpha} z_{\alpha}^{*}}-i g\left(z_{\alpha}-z_{\alpha}^{*}\right)\right] \\
&+\frac{\beta}{2}\left[\partial_{\alpha} \frac{\gamma^{2}}{z_{\alpha} z_{\alpha}^{*}}-A \gamma\left(\frac{q_{\alpha}}{z_{\alpha}}+\frac{q_{\alpha}^{*}}{z_{\alpha}^{*}}\right)\right]
\end{aligned}
$$

in which

$$
q^{*}=(2 \pi i)^{-1} P V \int_{-\infty}^{\infty} \frac{\gamma\left(\alpha^{\prime}, t\right)}{z(\alpha, t)-z\left(\alpha^{\prime}, t\right)} d \alpha^{\prime}
$$

and $A=\left(\rho_{+}-\rho_{-}\right) /\left(\rho_{+}+\rho_{-}\right)$is the Atwood number.

As before, $z \equiv \alpha$ and $\gamma(\alpha) \equiv 0$ is an equilibrium solution. Thus set

$$
\begin{aligned}
& z=\alpha+s=\alpha+s_{+}(\alpha, t)+s_{-}(\alpha, t), \\
& \gamma=\gamma_{+}(\alpha, t)+\gamma_{-}(\alpha, t)
\end{aligned}
$$

and define

$$
\begin{aligned}
& M_{1}[s, \gamma]=R_{1}[z, \gamma], \\
& M_{2}\left[s, s^{*}, \gamma\right]=R_{2}\left[z, z^{*}, \gamma\right] .
\end{aligned}
$$

Apply the localized approximation method of $\S 2$ to obtain

$$
\begin{aligned}
& \partial_{t} s_{+}=M_{1}\left[s_{-}, \gamma_{-}\right]^{*}, \\
& \partial_{t} s_{-}^{*}=M_{1}\left[s_{+}, \gamma_{+}\right], \\
& \partial_{t} \gamma_{+}=M_{2}\left[s_{+}, s_{-}^{*}, \gamma_{+}\right], \\
& \partial_{t} \gamma_{-}^{*}=M_{2}\left[s_{-}, s_{+}^{*}, \gamma_{-}\right]^{*} .
\end{aligned}
$$

Using the localized approximation, the integral term $q$ in $M_{1}$ is calculated from (5.2) by contour deformation to be

$$
\begin{aligned}
& q_{+}=\frac{1}{2} \gamma_{-}^{*} z_{-\alpha}^{*-1}, \\
& q_{-}^{*}=-\frac{1}{2} \gamma_{+} z_{+\alpha}^{-1}
\end{aligned}
$$

in which $z_{+}=\alpha+s_{+}$and $z_{-}=\alpha+s_{-}$. Then

$$
\begin{aligned}
& M_{1}\left[s_{-}, \gamma_{-}\right]^{*}=\frac{\beta+1}{2} \gamma_{-}^{*} z_{-\alpha}^{*-1}, \\
& M_{1}\left[s_{+}, \gamma_{+}\right]=\frac{\beta-1}{2} \gamma_{+} z_{+\alpha}^{-1}
\end{aligned}
$$


and the localized approximation for the differential operator $M_{2}$ gives

$$
\begin{gathered}
M_{2}\left[s_{+}, s_{-}^{*}, \gamma_{+}\right]=A\left[q_{-t}^{*} z_{+\alpha}+q_{+t} z_{-\alpha}^{*}+\frac{1}{4} \partial_{\alpha} \frac{\gamma_{+}^{2}}{z_{+\alpha} z_{-\alpha}^{*}}-i g\left(z_{+\alpha}-z_{-\alpha}^{*}\right)\right] \\
+\frac{\beta}{2}\left[\partial_{\alpha} \frac{\gamma_{+}^{2}}{z_{+\alpha} z_{-\alpha}^{*}}-A \gamma_{+}\left(\frac{q_{+\alpha}}{z_{+\alpha}}+\frac{q_{-\alpha}^{*}}{z_{-\alpha}^{*}}\right)\right] .
\end{gathered}
$$

Since the vortex strength $\gamma$ is real for $\alpha$ real, the $\gamma_{+}=\gamma_{-}^{*}$. Under this assumption the localized approximation equations (5.5)-(5.8) can be combined and simplified to obtain

$$
\begin{aligned}
& \partial_{t} s_{+}=\frac{\beta+1}{2}\left(1+s_{-\alpha}^{*}\right)^{-1} \gamma_{+}, \\
& \partial_{t} s_{-}^{*}=\frac{\beta-1}{2}\left(1+s_{+}\right)^{-1} \gamma_{+}, \\
& \partial_{t} \gamma_{+}=\frac{\beta+A}{2} \partial_{\alpha}\left[\left(1+s_{+\alpha}\right)^{-1}\left(1+s_{-\alpha}^{*}\right)^{-1} \gamma_{+}^{2}\right]-i A g \partial_{\alpha}\left(s_{+}-s_{-}^{*}\right) .
\end{aligned}
$$

Differentiate these equations in $\alpha$ and denote $\phi=\partial_{\alpha} s_{+}, \psi=\partial_{\alpha} s_{-}^{*}$, and $\omega=\gamma_{+}$. The result is the quasi-linear system

$$
\begin{aligned}
& \partial_{t} \phi=\frac{\beta+1}{2} \partial_{\alpha} \frac{\omega}{1+\psi}, \\
& \partial_{t} \psi=\frac{\beta-1}{2} \partial_{\alpha} \frac{\omega}{1+\phi}, \\
& \partial_{t} \omega=\frac{\beta+A}{2}\left[\partial_{\alpha} \frac{\omega^{2}}{(1+\phi)(1+\psi)}\right]-i A g(\phi-\psi) .
\end{aligned}
$$

The system (5.10) forms the localized approximation for the Rayleigh-Taylor problem. An analysis of this system by Siegel [18] shows that singularities form as expected, giving the first analytic prediction of singularities for the Rayleigh-Taylor problem. In addition, at Atwood number $A=1$, corresponding to liquid falling into a vacuum, two singularities of opposite sign coalesce and partially cancel. Such cancellation was conjectured by Baker, Meiron, and Orszag [1]. For this problem it is natural to take $\beta=-1$, so that the interface velocity is that of the liquid. Computation of singularity formation for the Rayleigh-Taylor problem in the Boussineq limit $(A \rightarrow 0$, $A g \rightarrow 1$ ) has been performed by Pugh [14], and the singularity time predicted by Siegel (for $\beta=0$ ) agrees well with Pugh's computations.

6. Desingularized vortex sheet equations. A desingularized approximation of the vortex sheet equations (3.1) was formulated by Krasny [9] and used by him to compute roll-up of a vortex sheet after singularity formation. Krasny's desingularized equation is

$$
\begin{aligned}
\partial_{t} \overline{z(\Gamma)} & =K_{\delta}[z, \bar{z}] \equiv(2 \pi i)^{-1} \int_{-\infty}^{\infty} \frac{\overline{z(\Gamma)-z\left(\Gamma^{\prime}\right)}}{\left|z(\Gamma)-z\left(\Gamma^{\prime}\right)\right|^{2}+\delta^{2}} d \Gamma^{\prime} \\
& =(2 \pi i)^{-1} \int_{-\infty}^{\infty} \frac{z^{*}-z^{* \prime}}{\left(z-z^{\prime}\right)\left(z^{*}-z^{* \prime}\right)+\delta^{2}} d \Gamma^{\prime} .
\end{aligned}
$$


As before set $z=\Gamma+s$ and $M_{\delta}\left[s, s^{*}\right]=K_{\delta}\left[z, z^{*}\right]$. The localized approximation for $(6.1)$ is

$$
\begin{aligned}
& \partial_{t} s_{+}=M_{\delta}\left[s_{-}, s_{+}^{*}\right]^{*}, \\
& \partial_{t} s_{-}^{*}=M_{\delta}\left[s_{+}, s_{-}^{*}\right] .
\end{aligned}
$$

Now calculate $M_{\delta}\left[s_{+}, s_{-}^{*}\right]$ and $M_{\delta}\left[s_{-}, s_{+}^{*}\right]^{*}$ for small $\delta$. The first of these is

$$
M_{\delta}\left[s_{+}, s_{-}^{*}\right]=-(2 \pi i)^{-1} \int_{-\infty}^{\infty} \frac{z_{-}^{* \prime}-z_{-}^{*}}{\left(z_{+}^{\prime}-z_{+}\right)\left(z_{-}^{* \prime}-z_{-}^{*}\right)+\delta^{2}} d \Gamma^{\prime}
$$

in which we denote $z_{+}=\Gamma+s_{+}(\Gamma), z_{-}^{*}=\Gamma+s_{-}^{*}(\Gamma), z_{+}^{\prime}=\Gamma^{\prime}+s_{+}\left(\Gamma^{\prime}\right)$, etc. The denominator is approximately

$$
\left(1+s_{+\Gamma}\right)\left(1+s_{-}^{*}\right)\left(\Gamma^{\prime}-\Gamma\right)^{2}+\delta^{2}
$$

so that it has poles at

$$
\Gamma^{\prime}=\Gamma_{ \pm}^{\prime}=\Gamma \pm i \delta\left(1+s_{+\Gamma}\right)^{-1 / 2}\left(1+s_{-\Gamma}^{*}\right)^{-1 / 2} .
$$

In computing the integral (6.3), the contour is deformed upwards so that the residue at $\Gamma_{+}^{\prime}$ is obtained and there is also a contribution of $\frac{1}{2}$ from the integral at $\infty$. Thus

$$
\begin{aligned}
M_{\delta}\left[s_{+}, s_{-}^{*}\right] & =-\underset{\Gamma^{\prime}=\Gamma_{+}^{\prime}}{\operatorname{res}}\left(\frac{z_{-}^{* \prime}-z_{-}^{*}}{\left(z_{+}^{\prime}-z_{+}\right)\left(z_{-}^{* \prime}-z_{-}^{*}\right)+\delta^{2}}\right)+\frac{1}{2} \\
& =-\frac{z_{-}^{* \prime}-z_{-}^{*}}{z_{+\Gamma}^{\prime}\left(z_{-}^{* \prime}-z_{-}^{*}\right)+z_{-\Gamma}^{* \prime}\left(z_{+}^{\prime}-z_{+}\right)}+\frac{1}{2} .
\end{aligned}
$$

Now expand this to get the leading order corrections in $\delta$, using the notation $\xi=\Gamma_{+}^{\prime}-\Gamma=$ $i \delta\left(1+s_{+\Gamma}\right)^{-1 / 2}\left(1+s_{-\Gamma}^{*}\right)^{-1 / 2}$. The result is

$$
\begin{aligned}
M_{\delta}\left[s_{+}, s_{-}^{*}\right] & =\frac{1}{2}-\frac{1}{2 z_{+\Gamma}}+\frac{1}{8}\left(\frac{z_{-\Gamma \Gamma}^{*}}{z_{+\Gamma} z_{-\Gamma}^{*}}+3 \frac{z_{+\Gamma \Gamma}}{z_{+\Gamma}^{2}}\right) \xi \\
& =\frac{1}{2} \frac{s_{+\Gamma}}{1+s_{+\Gamma}}+\frac{i \delta}{8}\left(\frac{s_{-\Gamma \Gamma}^{*}}{\left(1+s_{+\Gamma}\right)^{3 / 2}\left(1+s_{-\Gamma}^{*}\right)^{3 / 2}}+\frac{3 s_{+\Gamma \Gamma}}{\left(1+s_{+\Gamma}\right)^{5 / 2}\left(1+s_{-\Gamma}^{*}\right)^{1 / 2}}\right) .
\end{aligned}
$$

Similarly,

$$
M_{\delta}\left[s_{-}, s_{+}^{*}\right]^{*}=-\frac{1}{2} \frac{s_{-\Gamma}^{*}}{1+s_{-\Gamma}^{*}}-\frac{i \delta}{8}\left(\frac{s_{+\Gamma \Gamma}}{\left(1+s_{-\Gamma}^{*}\right)^{3 / 2}\left(1+s_{+\Gamma}\right)^{3 / 2}}+\frac{3 s_{-\Gamma \Gamma}^{*}}{\left(1+s_{-\Gamma}^{*}\right)^{5 / 2}\left(1+s_{+\Gamma}\right)^{1 / 2}}\right)
$$

These expressions may be substituted into (6.2) to obtain the localized approximation for Krasny's desingularized vortex sheet equation.

Krasny's desingularized equation can be compared to the equation for motion of vortex layer of small thickness derived by Moore [10] and studied by Baker and Shelley [2]. Let $\varepsilon=H / \rho$ be the dimensionless measure of thickness for the layer, with $H$ the dimensional thickness and $\rho$ the radius of curvature. Let $\bar{\omega}$ be the (constant) vorticity in the layer, so that $\omega=\varepsilon \bar{\omega}$ is the corresponding vortex sheet strength and is assumed to be $O(1)$ in size. Then the equation of motion for the center line $z(\Gamma, t)=\Gamma+s_{+}+s_{-}$ of the vortex layer is

$$
\partial_{t} \bar{z}=M_{\varepsilon}\left[s^{\prime}, s^{*}\right] \equiv(2 \pi i)^{-1} \int\left(z(\Gamma)-z\left(\Gamma^{\prime}\right)\right)^{-1} d \Gamma^{\prime}+\varepsilon(6 \omega i)^{-1} \partial_{\Gamma}\left(\left|z_{\Gamma}\right|^{-4} \bar{z}_{\Gamma}\right)
$$


in which terms of size $O\left(\varepsilon^{2}\right)$ have been ignored. The localized approximation for (6.9) is

$$
\begin{aligned}
& \partial_{t} s_{+}=M_{\varepsilon}\left[s_{-}, s_{+}^{*}\right]^{*}=-\frac{1}{2} s_{-\Gamma}^{*}\left(1+s_{-\Gamma}^{*}\right)^{-1}-\varepsilon(6 \omega i)^{-1} \partial_{\Gamma}\left(\left(1+s_{-\Gamma}^{*}\right)^{-2}\left(1+s_{+\Gamma}\right)^{-1}\right), \\
& \partial_{t} s_{-}^{*}=M_{\varepsilon}\left[s_{+}, s_{-}^{*}\right]=\frac{1}{2} s_{+\Gamma}\left(1+s_{+\Gamma}\right)^{-1}+\varepsilon(6 \omega i)^{-1} \partial_{\Gamma}\left(\left(1+s_{+\Gamma}\right)^{-2}\left(1+s_{-\Gamma}^{*}\right)^{-1}\right) .
\end{aligned}
$$

Comparison of (6.8) and (6.10) shows them to be significantly different so that the desingularization parameter $\delta$ cannot be interpreted as vortex layer thickness. This difference can be understood by noting that Moore's expansion parameter is $(\varepsilon / \omega)$ which has units of (length/velocity). A more physically meaningful desingularization is found by replacing $\delta^{2}$ in $(6.1)$ by $(\varepsilon / \omega)^{2}$. Then a factor with units of velocity must be put into the first term of the denominator. A natural choice is $\left|z_{\Gamma}\right|^{2}$ or $\left|z_{\Gamma}^{\prime}\right|^{2}$ (note that the circulation variable $\Gamma$ has units of velocity $\times$ length).

Consider the desingularized integral

$$
\begin{aligned}
N_{\varepsilon}\left[s, s^{*}\right] & =(2 \pi i)^{-1} \int_{-\infty}^{\infty} \frac{\overline{\left(z-z^{\prime}\right)}\left(\alpha\left|z_{\Gamma}\right|^{2}+\beta\left|z_{\Gamma}^{\prime}\right|^{2}\right)}{\left|z-z^{\prime}\right|^{2}\left(\alpha\left|z_{\Gamma}\right|^{2}+\beta\left|z_{\Gamma}^{\prime}\right|^{2}\right)+(\varepsilon / \omega)^{2}} d \Gamma^{\prime} \\
& =(2 \pi i)^{-1} \int_{-\infty}^{\infty} \frac{\left(z^{*}-z^{* \prime}\right)\left(\alpha z_{\Gamma} z_{\Gamma}^{*}+\beta z_{\Gamma}^{\prime} z_{\Gamma}^{* \prime}\right)}{\left(z-z^{\prime}\right)\left(z^{*}-z^{* \prime}\right)\left(\alpha z_{\Gamma} z_{\Gamma}^{*}+\beta z_{\Gamma}^{\prime} z_{\Gamma}^{* \prime}\right)+(\varepsilon / \omega)^{2}} d \Gamma^{\prime} .
\end{aligned}
$$

The localized approximation for $N_{\varepsilon}$ is

$$
N_{\varepsilon}\left[s, s^{*}\right] \cong N_{\varepsilon}\left[s_{+}, s_{-}^{*}\right]+N_{\varepsilon}\left[s_{-}, s_{+}^{*}\right] .
$$

The first of these is evaluated by the same procedure as for Krasny's desingularized integral to obtain

$$
\begin{aligned}
N_{\varepsilon}\left[s_{+}, s_{-}^{*}\right] \cong \frac{1}{2} & \frac{s_{+\Gamma}}{1+s_{+\Gamma}}+\frac{i}{8}\left(\frac{\varepsilon}{\omega}\right)(\alpha+\beta)^{-3 / 2} \\
& \cdot\left\{(\alpha+3 \beta) \frac{s_{-\Gamma \Gamma}^{*}}{\left(1+s_{+\Gamma}\right)^{2}\left(1+s_{-\Gamma}^{*}\right)^{2}}+(3 \alpha+5 \beta) \frac{s_{+\Gamma \Gamma}}{\left(1+s_{+\Gamma}\right)^{3}\left(1+s_{-\Gamma}^{*}\right)}\right\} .
\end{aligned}
$$

This agrees with the vortex layer equations, i.e.,

$$
N_{\varepsilon}\left[s_{+}, s_{-}^{*}\right]=M_{\varepsilon}\left[s_{+}, s_{-}^{*}\right]+O\left(\frac{\varepsilon}{\omega}\right)^{2}
$$

if $\alpha=\beta=\frac{9}{8}$. Similarly, $N_{\varepsilon}\left[s_{-}, s_{+}^{*}\right]=M_{\varepsilon}\left[s_{-}, s_{+}^{*}\right]+O(\varepsilon / \omega)^{2}$. Therefore we propose the following physically desingularized vortex sheet equation:

$$
\partial_{t} \bar{z}=(2 \pi i)^{-1} \int_{-\infty}^{\infty} \frac{\left(\bar{z}-\bar{z}^{\prime}\right)\left(\left|z_{\Gamma}\right|^{2}+\left|z_{\Gamma}^{\prime}\right|^{2}\right)}{\left|z-z^{\prime}\right|^{2}\left(\left|z_{\Gamma}\right|^{2}+\left|z_{\Gamma}^{\prime}\right|^{2}\right)+\frac{8}{9}(\varepsilon / \omega)^{2}} d \Gamma^{\prime} .
$$

Numerical solutions of this equation are now being performed.

7. Conclusions. We have reformulated and generalized an approximation method of Moore and applied it to a variety of fluid flow problems involving vortex sheets. Other applications of the method to circular vortex sheets and ringwings have been made by Orellana [13]. We have also applied this localized approximation method to a slightly perturbed strip of constant vorticity but with a trivial result; the linearized equations are all that results. In general this approximation method is applicable to the motion of any front, if the motion of the front can be described by an integrodifferential equation along the front. 
An improved version of the localized approximation has been formulated by Caflisch and Semmes [5]. As described in $\S 2$, the present approximation method is valid only for $\rho \gg 1$, i.e., for singularities far from the physically meaningful real line. In [5] the approximation is altered to a more complicated form that is valid up to the time that singularities hit the real axis and hence appear physically. However we believe that this improved approximation does not give a qualitative change in the singularity formation.

\section{REFERENCES}

[1] G. R. BAKer, D. I. Meiron, ANd S. A. Orszag, Boundary integral methods for axisymmetric and three-dimensional Rayleigh-Taylor instability problems, Phys. D, 12 (1984), pp. 19-31.

[2] G. R. BAKER AND M. SHelley, On the connection between thin vortex layers and vortex sheets. Part I. Asymptotic analysis; Part II. Numerical study, J. Fluid Mech., to appear.

[3] G. BIRKHOFF, Helmholtz and Taylor instability in hydrodynamic instability, in Proc. Symposium on Applied Mathematics XII, American Mathematical Society, Providence, RI, 1962, pp. 55-76.

[4] R. CAFlisCH AND O. OREllanA, Long time existence for a slightly perturbed vortex sheet, Comm. Pure Appl. Math., 39 (1986), pp. 807-838.

[5] R. CAFLISCH AND S. SEMmes, A nonlinear approximation for vortex sheet evolution and singularity formation, Phys. D, to appear.

[6] R. CAFLISCH, N. ERCOLANI, AND T. HOU, Singularity formation on planar vortex sheets, in preparation.

[7] S. D. Howison, J. R. OCKEndon, AND A. A. LACEY, Singularity development in moving-boundary problems, Quart. J. Mech. Appl. Math., 38 (1985), pp. 343-360.

[8] R. KRASNy, On singularity formation in a vortex sheet and the point vortex approximation, J. Fluid Mech., 167 (1986), pp. 65-93.

[9] — Desingularization of periodic vortex sheet roll-up, J. Comput. Phys., 65 (1986), pp. 292-313.

[10] D. W. MoORE, The equation of motion of a vortex layer of small thickness, Stud. Appl. Math., 58 (1978), pp. 119-140.

[11] - The spontaneous appearance of a singularity in the shape of an evolving vortex sheet, Proc. Roy. Soc. London Ser. A, 365 (1979), pp. 105-119.

[12] - Numerical and analytical aspects of Helmholtz instability in theoretical and applied mechanics, in Proc. XVI ICTAM, F. Niordson and N. Olhoff, eds., North-Holland, Amsterdam, 1984, pp. 629633.

[13] O. Orellana, Approximate theory for the nonlinear evolution of vortex sheets, Ph.D. thesis, New York University, New York, 1986.

[14] D. Pugh, Development of vortex sheets in Boussinesq flows-Formation of singularities, Ph.D. thesis, Imperial College, 1989.

[15] P. G. SAFFMAN, Exact solutions for the growth of fingers from a flat interface between two fluids in a porous medium or Hele-Shaw cell, Quart. J. Mech. Appl. Math., 12 (1959), pp. 146-150.

[16] P. G. SAFFMAN AND G. I. TAYLOR, The penetration of a fluid into a porous medium or Hele-Shaw cell containing a more viscous liquid, Proc. Roy. Soc. London Ser. A, 245 (1958), pp. 312-329.

[17] M. SHelley, A study of singularity formation in vortex sheet motion by a spectral accurate method, J. Fluid Mech., submitted.

[18] M. SIEGEL, An analytical and numerical study of singularity formation in the Rayleigh-Taylor problem, Ph.D. thesis, New York University, New York, 1989.

[19] G. TrygVasson And H. Aref, Numerical experiments on Hele-Shaw flow with a sharp interface, J. Fluid Mech., 136 (1983), pp. 1-30. 